\title{
The impact of pulse duration on multiphoton ionization in the soft $X$-ray regime
}

\author{
Mathias Richter*a ${ }^{\mathrm{a}}$, Andrey A. Sorokin ${ }^{\mathrm{b}}$, Kai Tiedtke $^{\mathrm{b}}$, \\ ${ }^{a}$ Physikalisch-Technische Bundesanstalt (PTB), Abbestr. 2-12, 10587 Berlin, Germany \\ ${ }^{\mathrm{b}}$ Deutsches Elektronen-Synchrotron (DESY), Notkestr. 85, 22607 Hamburg, Germany
}

\begin{abstract}
At the soft X-ray free electron laser FLASH, multiphoton ionization of free atoms has been studied by ion time-of-flight spectroscopy. Depending on the multiphoton mechanism, the ionization processes are influenced in different ways by the FEL pulse duration. This feature has been used, e.g., to measure the pulse duration of FLASH in the femtosecond regime by non-linear autocorrelation. In the present contribution, the impact of pulse duration on multiphoton ionization is discussed with an emphasis on the distinction between sequential and non-sequential processes, and collective electron excitation as well.
\end{abstract}

Keywords: non-linear processes, multiphoton ionization, gas-phase experiments, ion time-of-flight spectroscopy, FEL pulse duration

\section{INTRODUCTION}

The advent of the new X-ray free electron laser (FEL) facilities has considerably extended the field of non-linear photonmatter interaction towards higher photon energies [1-7]. With photon pulse energies of hundreds of microjoules and pulse durations in the femtosecond regime, different types of multiphoton ionization have meanwhile been studied on atoms, molecules, and clusters in the soft and hard X-ray regime [6-34]. In contrast to optical radiation, here, the photon energy exceeds the first ionization thresholds so that sequences of one-photon processes may occur, in which an ion created in a preceding photoionization step represents a new target for a subsequent step. Sequential processes even dominate non-linear photoionization with X-rays. Non-sequential, i.e. simultaneous multiphoton ionization and strongfield effects generally play a minor role.

However, not all non-linear photoionization phenomena studied in the X-ray range have so far been completely understood. A prominent example represents the multiphoton multiple ionization of Xe atoms in the photon energy range of the so-called Xe $4 d$ giant resonance. In the extreme ultraviolet (EUV) at about $93 \mathrm{eV}$ photon energy, charge states of up to $\mathrm{Xe}^{21+}$ were observed at irradiance levels of the order of $10^{16} \mathrm{~W} \mathrm{~cm}^{-2}$ [13]. The results are controversially discussed in terms of sequential photoionization schemes $[35,36]$ and collective electron excitation by the radiation field $[20,37$, 38].

In the present paper, the different schemes of non-linear photoionization and corresponding examples of the results of ion time-of-flight (TOF) spectroscopy obtained at the soft X-ray FEL facility FLASH are discussed in terms of the photon pulse duration. The latter influences the photoionization rates in different ways, depending on the mechanism at the rear. As a consequence, the distinction between different multiphoton ionization schemes may be realized by experimental investigations at varying pulse duration.

*mathias.richter@ptb.de; phone +49 30 3481-7100; fax +49 303481 7102; ptb.de

Advances in X-ray Free-Electron Lasers II: Instrumentation, edited by

Thomas Tschentscher, Kai Tiedtke, Proc. of SPIE Vol. 8778, 877808 · C 2013

SPIE - CCC code: 0277-786X/13/\$18 · doi: 10.1117/12.2021298 


\section{EXPERIMENT}

For our ion TOF experiments, we applied the focusing geometries shown in figure 1 [37]. For the measurements at 42.8 $\mathrm{eV}$ photon energy, the focus of the beamline BL2 at FLASH was used (figure 1A) with a FWHM diameter of $15 \mu \mathrm{m}$ [39]. The FEL beam was introduced into a homogeneous gas target at pressures from $10^{-4}$ to $10^{-3} \mathrm{~Pa}$. Generated photoions were extracted towards the TOF spectrometer entrance aperture by a homogeneous electric field. The aperture width of $1 \mathrm{~mm}$ along the beam ensured photoion collection exclusively from the focus region with a Rayleigh length larger than $1 \mathrm{~mm}$. The ions were detected by means of an open multiplier whose linearity was tested in the range from 1 to $10^{6}$ ions per FEL pulse. The whole apparatus could be moved $\pm 2 \mathrm{~cm}$ along the FEL beam in order to vary the beam cross section and, hence, the mean irradiance within the interaction volume accepted by the TOF spectrometer.

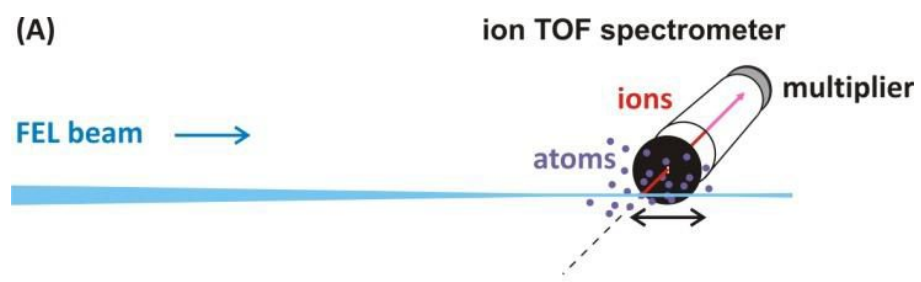

(B)

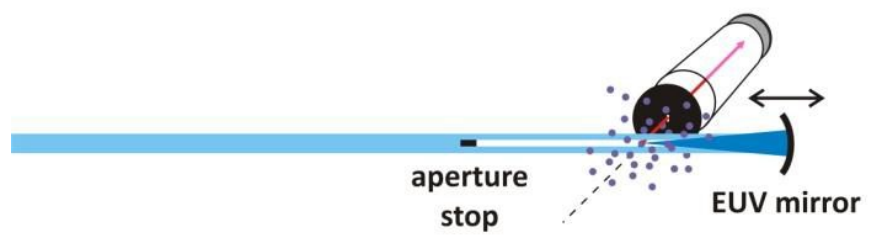

(C)

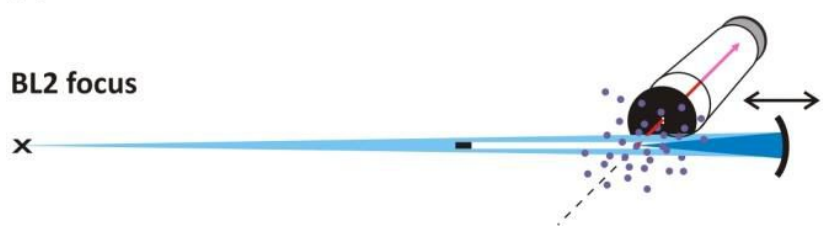

Figure 1. Schematic diagrams of the experimental set-ups as used for ion TOF spectroscopy on atoms at FLASH: (A) in the focus of beamline BL2; (B) in the focus of a spherical EUV multilayer mirror; (C) in the demagnified BL2 focus using a spherical EUV multilayer mirror [37].

In the extreme ultraviolet (EUV), the FEL beam was focused by spherical Mo-Si multilayer mirrors at a $20 \mathrm{~cm}$ distance from the TOF interaction volume in back-reflection geometry, as shown in figures 1B and 1C. A mirror reflectance in the order of $68 \%$ was achieved [40] and microfocuses of 3 to $5 \mu \mathrm{m}$ in diameter (FWHM) could be realized [13, 20]. As a result, the irradiance levels were higher by one to two orders of magnitude than during our measurements in the BL2 focus. In the first EUV measurement geometry, the FEL was directly focused (figure 1B), while in the second, the BL2 focus was demagnified by the multilayer mirror (figure 1C). In both cases, an aperture stop of $1.5 \mathrm{~mm}$ in height was used in combination with a spectrometer entrance aperture of $1.0 \mathrm{~mm}$ in height to prevent ion signals from the incident unfocused radiation. Moreover, the aperture width along the FEL beam was reduced, compared to the measurements in the BL2 focus, to about $0.3 \mathrm{~mm}$ to account for the much shorter Rayleigh length. Instead of the apparatus, the mirror was moved along the FEL beam to vary the beam cross section and the irradiance within the interaction volume.

For the measurement of the FEL beam cross sections in the micrometer regime, we applied the so-called target depletion effect [39] which originates from the successive reduction of target atoms within the interaction volume by photoionization processes within a single FEL pulse. FEL pulse energies in the $\mu \mathrm{J}$ regime were recorded in absolute terms by the use of calibrated gas-monitor detectors [41, 42]. FEL pulse duration was finally determined by applying a wave front split and delay unit $[43,44]$. 


\section{SIMULTANEOUS MULTIPHOTON IONIZATION}

The interaction of electromagnetic radiation with an atom is described by the time-dependent Schrödinger equation:

$$
i \hbar \frac{\partial}{\partial t} \Psi=H \Psi
$$

Here, $\Psi$ denotes the atomic wavefunction and $H$ the Hamiltonian operator. The latter consists of the unperturbed Hamiltonian of the stationary ground state of the atom $H_{0}$ and the time-dependent perturbation due to the electromagnetic field:

$$
H=H_{0}+\sum_{i}\left(\frac{-e}{m c} \vec{p}_{i} \vec{A}(t)+\frac{e^{2}}{2 m c^{2}}|\vec{A}(t)|^{2}\right)
$$

with the momentums $\vec{p}_{i}$ of the atomic electrons and the vector potential $\vec{A}$. The solution of this equation requires approximation. For a sufficiently small perturbation, low-order time-dependent perturbation theory may be applied which yields the general power law for the rate of $n$-photon processes [45]:

$$
\dot{N}^{(n)}=N_{0} \sigma^{(n)}\left(\frac{E}{\hbar \omega}\right)^{n}
$$

with the number of targets within the interaction volume $N_{0}$, the respective generalized $n$-photon ionization cross section $\sigma^{(n)}$, the photon energy $\hbar \omega$, and the irradiance $E$. The latter is often called 'intensity' and represents a key quantity of the radiation field to describe multiphoton processes. It is related to the fluence $F$, i.e. the number of photons per unit area $d N_{p h} / d A$, by:

$$
E=\dot{F} \hbar \omega
$$

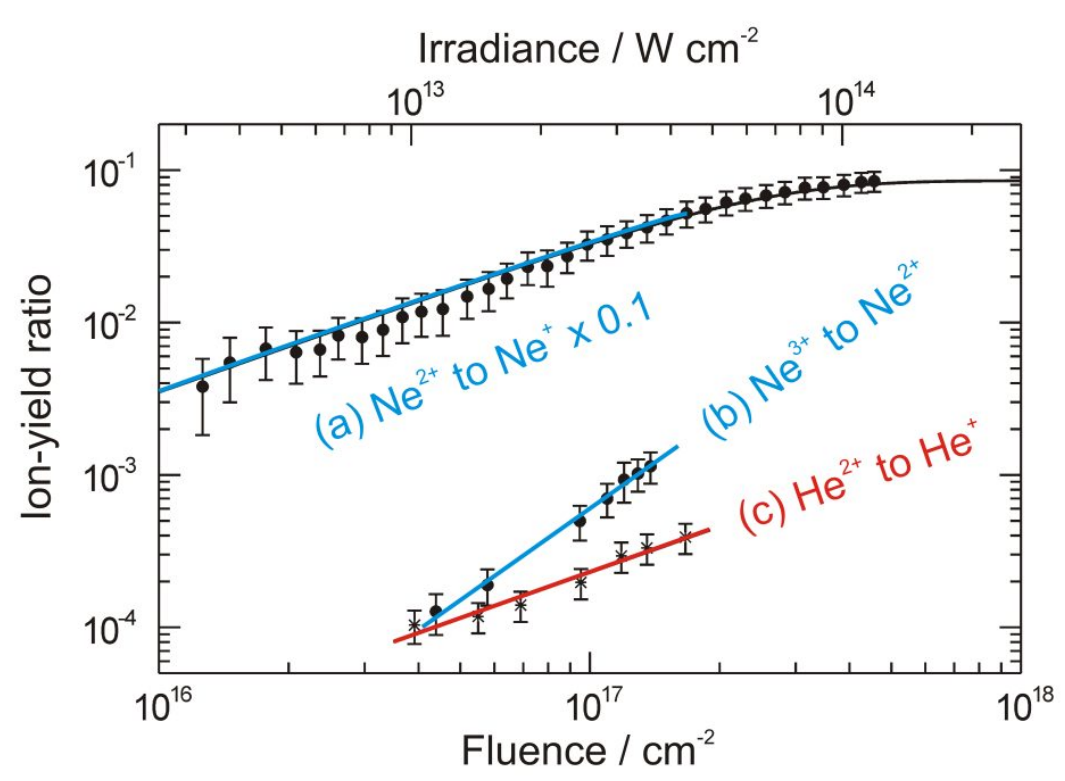

Figure 2. Ion yield ratios as a function of mean irradiance and fluence taken at $42.8 \mathrm{eV}$ photon energy: (a) $\mathrm{Ne}^{2+}$ to $\mathrm{Ne}^{+}$ratio, (b) $\mathrm{Ne}^{3+}$ to $\mathrm{Ne}^{2+}$ ratio, and (c) $\mathrm{He}^{2+}$ to $\mathrm{He}^{+}$ratio [14].

As a result, for a radiation pulse of constant fluence, the number of emerging $n$-photon processes decreases explicitly with the pulse duration $\Delta t$ by: 


$$
N^{(n)}=\Delta t N_{0} \sigma^{(n)}\left(\frac{F}{\Delta t}\right)^{n} \propto \Delta t^{1-n},
$$

i.e., with shorter pulse duration, the same number of photons per unit area results in more multiphoton $(n>2)$ processes.

In the (soft) X-ray regime, the power law of equation 3 was initially demonstrated using a high harmonics generation (HHG) source at the photon energy of $42 \mathrm{eV}$ [46] and later at FLASH at $42.8 \mathrm{eV} \mathrm{[14],} \mathrm{both} \mathrm{on} \mathrm{the} \mathrm{simultaneous} \mathrm{2-photon}$ double ionization of He. Figure $2 \mathrm{c}$ shows the corresponding linear increase of the $\mathrm{He}^{2+} / \mathrm{He}^{+}$ratio with the mean irradiance and the fluence of the FLASH pulses.

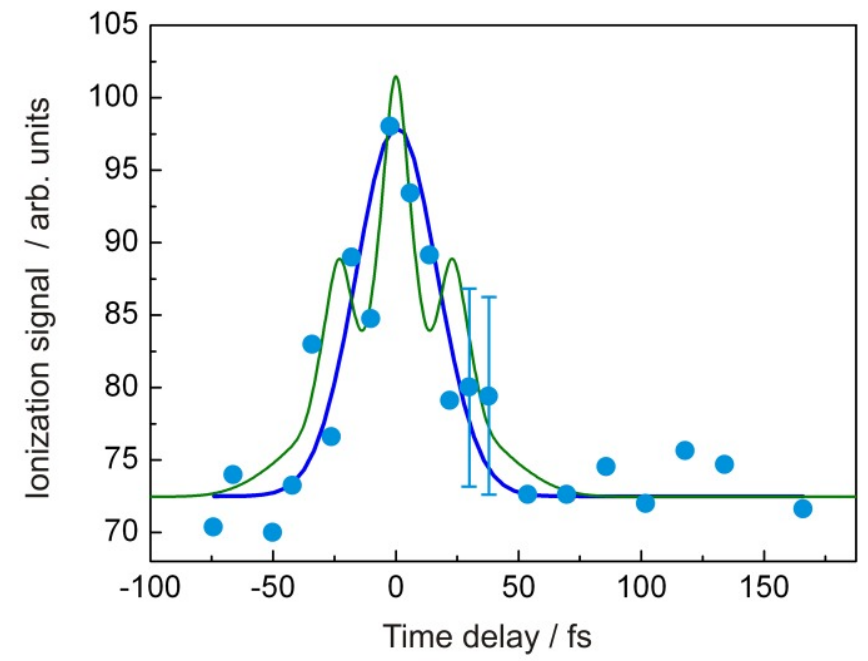

Figure 3. Double ionization signal of He measured at a photon energy of $51.8 \mathrm{eV}$ as a function of the temporal delay between two autocorrelator pulses (dots). The thick line represents a Gaussian function with $39 \mathrm{fs}$ FWHM yielding a pulse duration of $(28 \pm 5)$ fs. The thin line represents a simulation with a three-pulse structure for the temporal pulse shape of FLASH [44].

The quadratic dependence of the simultaneous 2-photon double ionization of $\mathrm{He}$ on the irradiance according to equation 3 has also been used to measure the pulse duration of FLASH via autocorrelation. By the use of a wavefront split and delay unit, the FEL beam with the temporal pulse shape $f(t)$ was split into two parts with the irradiance amplitudes $E_{1}$ and $E_{2}$ and a temporal delay of $t^{\prime}\left(E(t)=E_{1} f(t)+\mathrm{E}_{2} f\left(t-t^{\prime}\right)\right)$. With equation 3 and $n=2$, one obtains for the 2-photon double ionization intensity as a function of the temporal delay:

$$
\frac{N^{(2)}\left(t^{\prime}\right)}{N^{(2)}\left(t^{\prime} \rightarrow \infty\right)}-1 \propto \int_{-\infty}^{+\infty} f(t) f\left(t-t^{\prime}\right) d t,
$$

from which the pulse shape function $f(t)$ and, with that, the pulse duration were derived. Figure 3 shows the corresponding result obtained at $51.8 \mathrm{eV}$ photon energy [44]. The Gaussian fit to the data points yields an autocorrelation width of $39 \mathrm{fs}$ and the FLASH pulse duration of $(28 \pm 5) \mathrm{fs}$.

Together with the pulse energy $N_{p h} \hbar \omega$ and the beam cross section $A$, the knowledge of the pulse duration $\Delta t$ allows the mean pulse irradiance $E=\left(N_{p h} / A\right)(\hbar \omega / \Delta t)$ to be calculated in absolute terms and multiphoton cross sections as well. Using equation 3, the literature data of the He single ionization cross section $\left(\sigma^{(1)}\right)$ [47], and experimental $\mathrm{He}^{2+}$ to $\mathrm{He}^{+}$ ratios $\left(\dot{N}^{(2)} / \dot{N}^{(1)}\right)$ obtained at FLASH [14], the simultaneous 2-photon double ionization cross section of He was derived according to:

$$
\sigma^{(2)}=\sigma^{(1)} \frac{\dot{N}^{(2)}}{\dot{N}^{(1)}} \frac{\hbar \omega}{E}
$$


The result is shown in figure 4, together with the corresponding HHG result and some predictions. The simultaneous 2photon double ionization of $\mathrm{He}$ has been a showcase of considerable interest during the past few years, as is demonstrated by numerous theoretical papers (see, e.g., [48-67]).

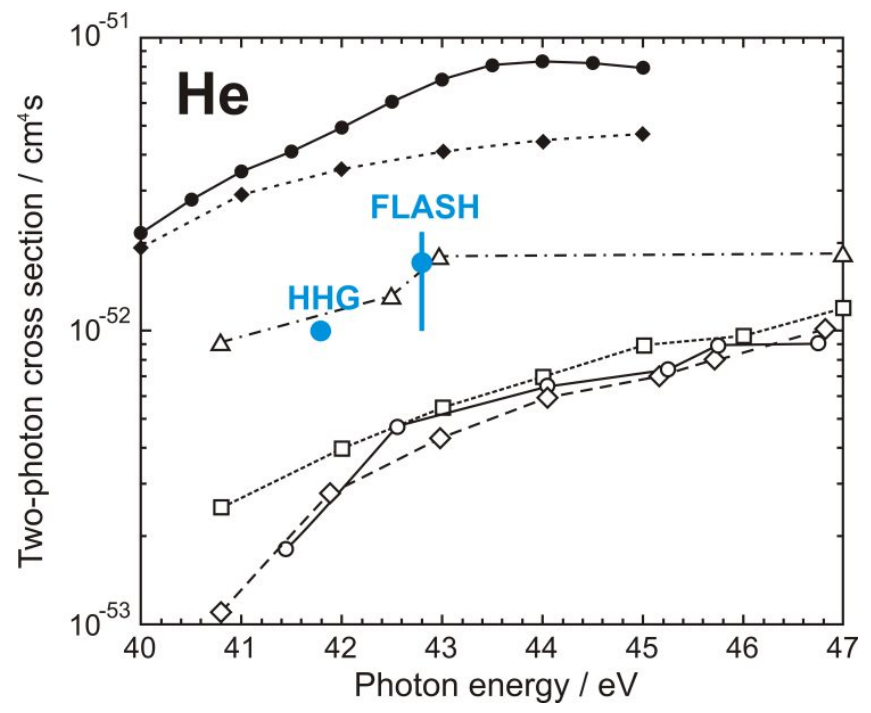

Figure 4. Two-photon double ionization cross section of He as measured at FLASH [14] and using an HHG source [46, 68]. Some theoretical predictions are shown for comparison: $\bullet \bullet$ [51], $\diamond \diamond$ [50], $\triangle \triangle \triangle$ [55], $\square \square \square$ [49], $\diamond \diamond \diamond$ [48], $\bigcirc \bigcirc \bigcirc[51]$.

In a similar way, the absolute cross section for simultaneous 2-photon single ionization of $\mathrm{Ne}^{2+}$ was derived from the $\mathrm{Ne}^{3+}$ to $\mathrm{Ne}^{2+}$ ratios in figure $2 \mathrm{~b}$, which has been found to be about 20 times larger at $42.8 \mathrm{eV}$ photon energy than the cross section for simultaneous 2-photon double ionization of $\mathrm{He}\left((3.0 \pm 1.2) \times 10^{-51} \mathrm{~cm}^{4} \mathrm{~s}\right.$ vs. $\left.(1.6 \pm 0.6) \times 10^{-52} \mathrm{~cm}^{4} \mathrm{~s}\right)$. Another very specific example of simultaneous 2-photon single ionization that was experimentally investigated in the soft X-ray regime is the $4 d$ above-threshold ionization (ATI) of Xe [28]. In the case of ATI, an electron absorbs more photons than required for ionization. In the present case, two-photon $4 d$ ionization of Xe was observed by electron spectroscopy at $93 \mathrm{eV}$ photon energy, i.e. about $25 \mathrm{eV}$ above the $4 d$ ionization threshold.

\section{IONIZATION SEQUENCES}

Simultaneous multiphoton ionization may be described by a sequence of excitations via virtual intermediate states as depicted in figure 5a. In case these intermediate states become real, sequential multiphoton ionization occurs in which an excited atom or ion created in a preceding step represents the target for a subsequent step (figure 5b). If the photon energy fits, resonance states may be populated. However, in the regular case, each step of such an ionization sequence leads to an increase in the charge state by one as long as the photon energy exceeds the respective ionization energy. Such a scheme may be described by a system of coupled rate equations which can be numerically solved [69]:

$$
\begin{aligned}
& \dot{N}_{0}(t)=-\sigma_{01} \dot{F}(t) N_{0}(t) \\
& \dot{N}_{1}(t)=\sigma_{01} \dot{F}(t) N_{0}(t)-\sigma_{12} \dot{F}(t) N_{1}(t) \\
& \dot{N}_{2}(t)=\sigma_{12} \dot{F}(t) N_{1}(t)-\sigma_{23} \dot{F}(t) N_{2}(t)
\end{aligned} .
$$




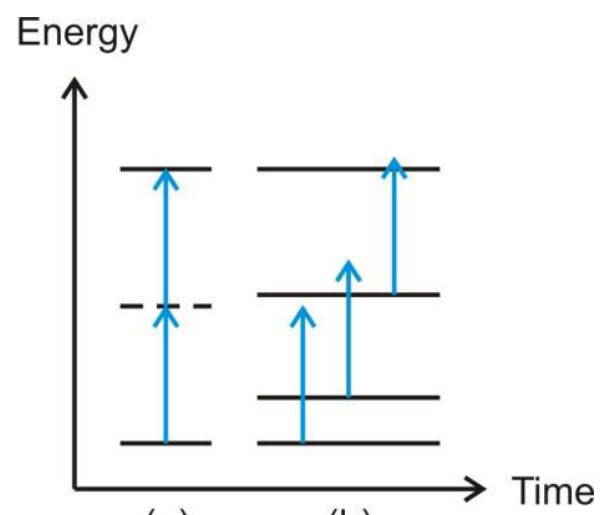

(a)

(b)

Figure 5. Energy level diagram and scheme for simultaneous 2-photon excitation via a virtual state (a) and sequential 3photon excitation via real states (b).

Here, $N_{i}$ denotes the number of species of charge state $i+, \sigma_{i j}$ the cross section for the step from $i+$ to $j+$, and $\dot{F}$ the photon flux. In contrast to simultaneous multiphoton processes (equation 5), the number of sequential $n$-photon processes per pulse does not explicitly depend on the pulse duration $\Delta t$ as long as $\Delta t$ is short enough, so that the atomic and ionic targets do not drop out of the interaction volume. This can also be directly seen from an analytical solution of the rate equation system of equation 8 with the simplification that the cross sections $\sigma_{i j}$ are equal $(=\sigma)$ for all $i j$ [70], which leads to a power law as a function of fluence $(F)$ instead of irradiance $(E)$ :

$$
N_{n}=N_{0} \frac{\sigma^{n} F^{n}}{n !} \exp (-\sigma F),
$$

i.e., in fact, without dependence on $\Delta t$. Generally, sequential multiphoton ionization is also stronger by orders of magnitude than simultaneous multiphoton ionization. For the $\mathrm{Ne} \rightarrow \mathrm{Ne}^{+} \rightarrow \mathrm{Ne}^{2+}$ sequence at $42.8 \mathrm{eV}$ photon energy, this can be seen from the strong $\mathrm{Ne}^{2+} / \mathrm{Ne}^{+}$ratio in figure $2 \mathrm{a}$ in comparison to the much weaker ratios of figure $2 \mathrm{~b}$ and figure $2 \mathrm{c}$ which correspond to simultaneous multiphoton processes.

However, particularly if inner-shell electrons are involved, decay and relaxation processes may compete with a further excitation/ionization step within an ionization sequence. In such cases, the pulse duration may influence to what extent decay and relaxation processes may occur. A prominent example represents the significant dependence of sequential multiphoton ionization of $\mathrm{Ne}$ on the FEL pulse duration as observed at the Linac Coherent Light Source (LCLS) at $2 \mathrm{keV}$ photon energy [26]. Here, the full stripping of the ten Ne electrons is explained by a combination of photoionization in the $1 s$ shell and Auger decay. This mechanism, however, requires sufficiently long laser pulses so that the $1 s$ shell may be refilled from time to time by an Auger process before the next photoionization step occurs.

\section{COLLECTIVE ELECTRON EXCITATION}

Another prominent example of multiphoton ionization in the soft X-ray and EUV regime represents the multiple ionization of $\mathrm{Xe}$ at about $93 \mathrm{eV}$ [13]. The generation of charge states of up to $\mathrm{Xe}^{21+}$ at irradiance levels of the order of $10^{16} \mathrm{~W} \mathrm{~cm}^{-2}$ may be explained by a sequential scheme which is shown in figure $6 \mathrm{a}$. Due to the so-called $4 d$ giant resonance in this photon energy regime (figure 7), the sequence starts with photoionization in the inner $4 d$ shell with subsequent Auger decay, in analogy to the Ne $1 s$ photoionization/Auger sequence [26] described at the end of the last section. For the higher steps $(>7+)$, however, more than one photon is required to reach the next charge state, respectively. As an example, for the $\mathrm{Xe}^{20+} \rightarrow \mathrm{Xe}^{21+}$ transition, at least seven EUV photons of $93 \mathrm{eV}$ are needed to be simultaneously absorbed. Altogether, in order to generate $\mathrm{Xe}^{21+}$ from atomic $\mathrm{Xe}$, more than $5 \mathrm{keV}$ energy, i.e. almost 60 EUV photons, must be absorbed by an individual atom within the estimated FLASH pulse duration during the experiments of about $15 \mathrm{fs}$. Hence, the scheme has to combine sequential and simultaneous multiphoton ionization in a complex manner. 

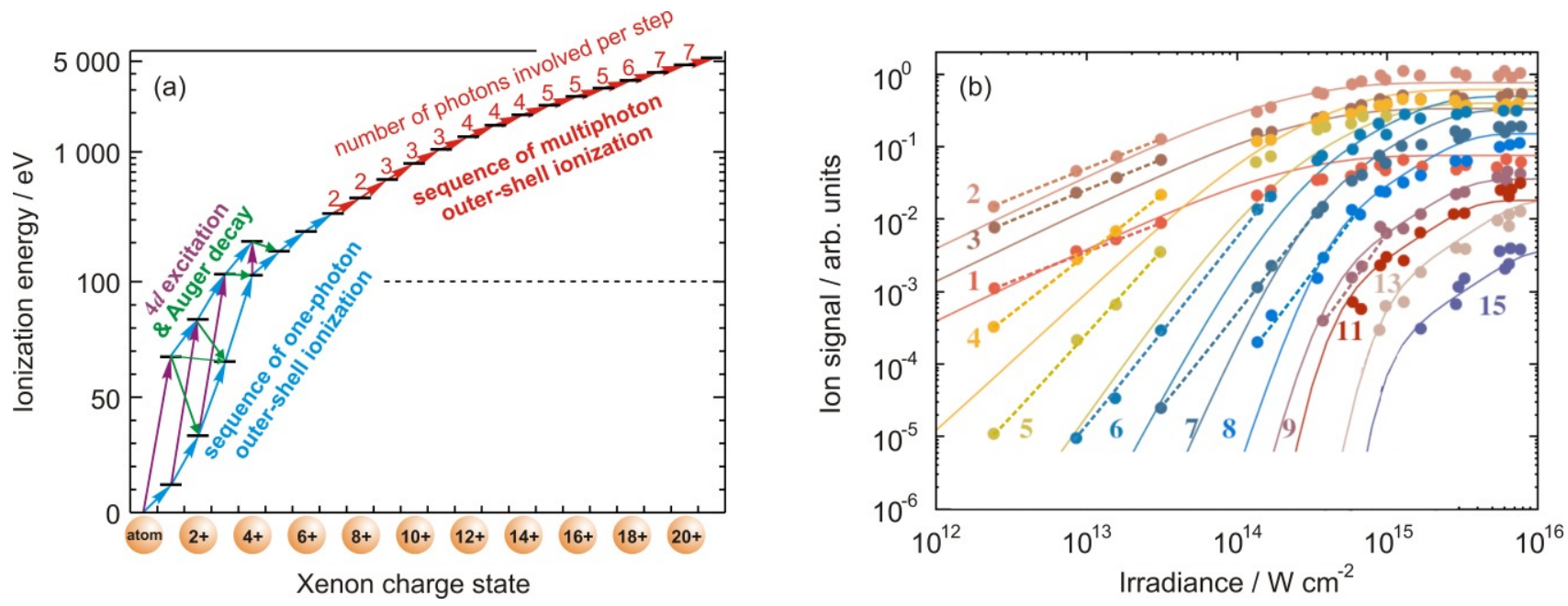

Figure 6. (a) Energy levels and sequential multiphoton ionization channels via ionic states for xenon at a photon energy of $93 \mathrm{eV}$ [13]. (b) Relative $\mathrm{Xe}^{q+}$ ion signal intensities as a function of the peak irradiance for different charge states $1 \leq q \leq 15$ at the photon energy of $93 \mathrm{eV}$ : the symbols show the experimental data obtained at FLASH [13], whose initial slopes are shown by the thick dashed lines (for $q \leq 9$ ); the thin solid lines represent model calculations within the framework of a sequential multiphoton scheme as shown in figure $6 \mathrm{a}[35,36]$.

The first theoretical study to explain the multiphoton multiple ionization of Xe by a sequential scheme resulted in the ion yields shown by the thin solid lines in figure $6 \mathrm{~b}[35,36]$. The approach had to consider at least 19 steps related to 19 coupled differential rate equations according to equation 8 with 19 individual one- or multiphoton ionization cross sections. Due to the complexity of the scheme, the cross sections were not calculated from first principles but obtained through a technique of scaling, with occasional adjustment to the experimental data.

Complementary to the approach of 60 individual photons interacting within 15 fs with more than 20 electrons of a single atom, also a collective model was proposed for the multiple ionization of Xe in the vicinity of the $4 d$ giant resonance. This strong and broad continuum resonance has, for many years, represented a prime example of the impact of electron correlation on inner-shell photoionization and has already been described by theories of collective electron oscillations in analogy to plasma excitations in a solid metal [71-74]. The simplest approximation for plasma oscillation, i.e. the damped harmonic oscillator, was used to reproduce the $4 d$ giant resonance cross section $\sigma$ (figure 7 ) and to express the energy transfer from a high-intensity EUV field to a single Xe atom [38]:

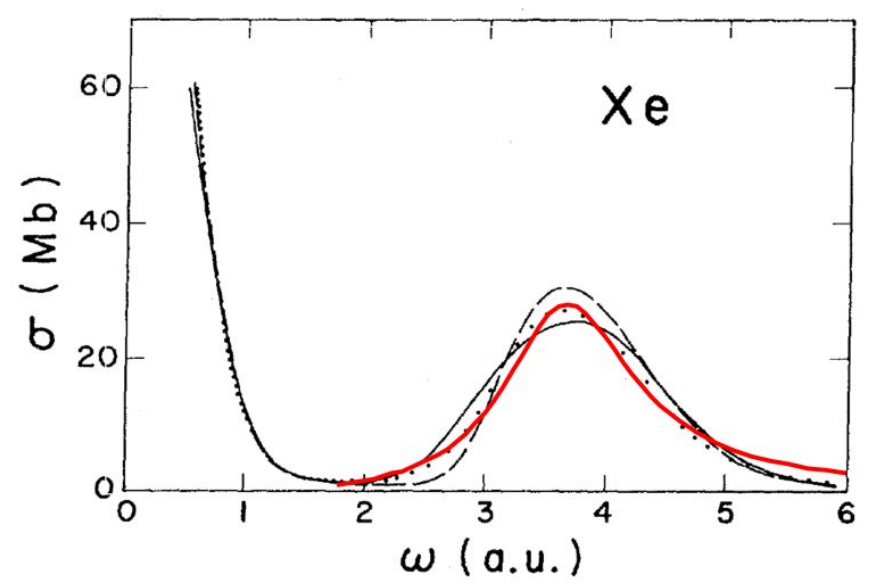

Figure 7. Photoabsorption cross section of Xe at the $4 d$ giant resonance (experimental data: points; relativistic timedependent local density approximation (RTDLDA): thin solid line; relativistic random phase approximation (RRPA): dashed line) [72]. The thick solid line shows the fitting result of a harmonic oscillator model [38]. 


$$
W \approx \sigma F \hbar \omega
$$

i.e. again without dependence on the pulse duration $\Delta t$. Within this model, the transferred energy is equal to the damping energy of the oscillator which leads to a sort of inner-atomic plasma heating and, based on a statistical electron energy distribution, to the emission of electrons.

\section{SUMMARY}

The interaction of X-ray laser pulses with matter at ultra-high intensities may be affected by various multiphoton excitation schemes which depend in different ways on the laser pulse duration. In the classical scheme of simultaneous multiphoton ionization, the same number of photons per unit area results in more processes at shorter pulse duration. Sequential schemes, on the other hand, which are restricted to photon energies above the respective ionization thresholds do not explicitly depend on the pulse duration. They may, however, be affected by the pulse duration dependence of decay and relaxation processes which are a part of them. The collective excitation of damped harmonic plasma oscillations of atomic electrons also depends just on the number of photons per unit area independently of the pulse duration. For future experiments at X-ray laser facilities, the different dependences on the laser pulse duration may be used to distinguish between the different multiphoton mechanisms. This would be of particular interest for the multiple ionization of Xe in the EUV, whose explanation still represents an unsolved problem of fundamental importance.

\section{REFERENCES}

[1] Ackermann, W. et al., Nat. Photonics 1, 336 (2007).

[2] Shintake, T. et al., Nat. Photonics 2, 555 (2008).

[3] Emma, P. et al., Nat. Photonics 4, 641 (2010).

[4] David, P. et al., Nat. Photonics 5, 456 (2011).

[5] Di Mitri, S. et al., Proc. IPAC 2011, 918 (2011).

[6] Bostedt, C. et al., Nucl. Instrum. Meth A 601, 108 (2009).

[7] Berrah, N. et al., J. Mod. Opt. 57, 1015 (2010).

[8] Wabnitz, H. et al., Nature 420, 482 (2002).

[9] Laarmann, T. et al., Phys. Rev. Lett. 92, 143401 (2004).

[10] Wabnitz, H. et al., Phys. Rev. Lett. 94, 23001 (2005).

[11] Sorokin, A. A. et al., J. Phys. B: At. Mol. Opt. Phys. B 39 L, 299 (2006).

[12] Moshammer, R. et al., Phys. Rev. Lett. 98, 203001 (2007).

[13] Sorokin, A. A. et al., Phys. Rev. Lett. 99, 213002 (2007).

[14] Sorokin, A. A. et al., Phys. Rev. A 75, 051402(R) (2007).

[15] Nagasono, M. et al., Phys. Rev. A 75, 051406(R) (2007).

[16] Bostedt, C. et al., Phys. Rev. Lett. 100, 133401 (2008).

[17] Rudenko, A. et al., Phys. Rev. Lett. 101, 073003 (2008).

[18] Sato, T. et al., Appl. Phys. Lett. 92, 15410 (2008).

[19] Jiang, Y. H. et al., Phys. Rev. Lett. 102, 123002 (2009).

[20] Richter, M. et al., Phys. Rev. Lett. 102, 163002 (2009).

[21] Fukuzawa, H. et al., Phys. Rev. A 79, 031201(R) (2009).

[22] Martins, M. et al., Phys. Rev. A 80, 023411 (2009).

[23] Jiang, Y. H. et al., J. Phys. B: At. Mol. Opt. Phys. B 42, 134012 (2009).

[24] Kurka, M. et al., J. Phys. B: At. Mol. Opt. Phys. B 42, 141002 (2009).

[25] Motomura, K. et al., J. Phys. B: At. Mol. Opt. Phys. B 42, 221003 (2009).

[26] Young, L. et al., Nature 466, 56 (2010).

[27] Meyer, M. et al., Phys. Rev. Lett. 104, 213001 (2010).

[28] Richardson, V. et al., Phys. Rev. Lett. 105, 013001 (2010).

[29] Hikosaka, Y. et al., Phys. Rev. Lett. 105, 133001 (2010). 
[30] Doumy, G. et al., Phys. Rev. Lett. 106, 083002 (2011).

[31] Kanter, E. P. et al., Phys. Rev. Lett. 107, 233001 (2011).

[32] Rohringer, N.et al., Nature 481, 488 (2012).

[33] Rudek, B. et al., Nat. Photonics 6, 857 (2012).

[34] Richardson, V. et al., J. Phys. B: At. Mol. Opt. Phys. 45, 085601 (2012).

[35] Makris, M. G. et al., Phys. Rev. Lett. 102, 033002 (2009).

[36] Lambropoulos, P. et al., J. Phys. B: At. Mol. Opt. Phys. 44, 175402 (2011).

[37] Richter, M. et al., J. Phys. B: At. Mol. Opt. Phys. 43, 194005 (2010).

[38] Richter, M., J. Phys. B: At. Mol. Opt. Phys. 44, 075601 (2011).

[39] Sorokin, A. A. et al., Appl. Phys. Lett. 89, 221114 (2006).

[40] Feigl, T., Yulin, S., Benoit, N. and Kaiser, N., Microelectron. Eng. 83, 703 (2006).

[41] Richter, M. et al., Appl. Phys. Lett. 83, 2970 (2003).

[42] Tiedtke, K. et al., J. Appl. Phys. 103, 094511 (2008).

[43] Mitzner, R. et al., Opt. Express 16, 19909 (2008).

[44] Mitzner, R. et al., Phys. Rev. A 80, 025402 (2009).

[45] Lambropoulos, P., Adv. At. Mol. Phys. 12, 87 (1976).

[46] Nabekawa, Y. et al., Phys. Rev. Lett. 94, 043001 (2005).

[47] Bizau, J.M. and Wuilleumier, F. J., J. Electron Spectrosc. Relat.Phenom. 71, 205 (1995).

[48] Feng, L. and van der Hart, H. W., J. Phys. B: At. Mol. Opt. Phys. B 36 L, 1 (2003).

[49] Laulan, S. and Bachau, H., Phys. Rev. A 68, 013409 (2003).

[50] Foumouo, E., Kamta, G. L., Edah, G. and Piraux, B., Phys. Rev. A 74, 063409 (2006).

[51] Nikolopoulos, L. A. A. and Lambropoulos, P., J. Phys. B: At. Mol. Opt. Phys. B 40, 1347 (2007).

[52] Horner, D. A. et al., Phys. Rev. A 76, 030701(R) (2007).

[53] Ivanov, I. A. and Kheifets, A. S., Phys. Rev. A 75, 033411 (2008).

[54] Feist, J. et al., Phys. Rev. A 77, 043420 (2008).

[55] Ivanov, I. A. and Kheifets, A. S., J. Phys. B: At. Mol. Opt. Phys. 41, 095002 (2008).

[56] Piraux, B., Foumouo, E., Antoine, P. and Bachau, H., J. Phys. Conf. Ser. 141, 012013 (2008).

[57] Foumouo, E., Antoine, P., Bachau, H. and Piraux, B., New J. Phys. 10, 025017 (2008).

[58] Palacios, A., Rescigno, T. N. and McCurdy, C. W., Phys. Rev. A 79, 033402 (2009).

[59] Førre, M., Selstø, S. and Nepstad, R., Phys. Rev. Lett. 105, 163001 (2010).

[60] Nepstad, R., Birkeland, T. and Førre, M., Phys. Rev. A 81, 063402 (2010).

[61] Foumouo, E. et al., J. Phys. B: At. Mol. Opt. Phys. 43, 091001 (2010).

[62] Zhang, Z., Peng, L.-Y., Gong, Q, and Morishita, T., Opt. Express 18, 8976 (2010).

[63] Pazourek, R. et al., Phys. Rev. A 83, 053418 (2011).

[64] Zhang, Z. et al., Phys. Rev. A 84, 043409 (2011).

[65] Selst $\varnothing$, S. et al., J. Phys. B: At. Mol. Opt. Phys. 44, 215003 (2011).

[66] Askeland, S., Nepstad, R. and Førre, M., Phys. Rev. A 85, 035404 (2012).

[67] Malegat, L., Bachau, H., Piraux, B. and Reynal, F., J. Phys. B: At. Mol. Opt. Phys. 45, 175601 (2012).

[68] Hasegawa, H. et al., Phys. Rev. A 71, 023407 (2005).

[69] Lambropoulos, P. et al., J. Opt. Soc. Am. B 4, 821 (1987).

[70] Guichard, R. et al., accepted for publication in J. Phys. B: At. Mol. Opt. Phys. (2013).

[71] Fano, U. and Cooper, J. W., Rev. Mod. Phys. 40, 441 (1968).

[72] Parpia, F. A., Johnson, W. R. and Radojević, V., Phys. Rev. A 29, 3173 (1968).

[73] Connerade, J. P., Esteva, J. M. and Karnatak R. C., "Giant Resonances in Atoms, Molecules, and Solids", New York Plenum (1987).

[74] Amusia, M. Ya. and Connerade, J. P., Rep. Prog. Phys. 63, 41 (2000). 